\title{
Las prácticas de copresencia durante la COVID-19: co-playing en plataformas online
}

\author{
Fernanda PIRES \\ Universitat Pompeu Fabra \\ fernanda.pires@upf.edu \\ https://orcid.org/0000-0001-6172-7594
}

\author{
Júlia VILAsís-Pamos \\ Universitat Pompeu Fabra \\ julia.vilasis@upf.edu \\ https://orcid.org/0000-0002-2060-4544
}

\author{
Priscila Álvarez-Cueva \\ Universitat Pompeu Fabra \\ priscila.alvarez@upf.edu \\ https://orcid.org/0000-0002-7570-6548
}

\author{
Ona Anglada-Pujol \\ Universitat Pompeu Fabra \\ ona.anglada@upf.edu \\ https://orcid.org/0000-0003-3078-7740
}

\section{Co-presence practices during COVID-19: co-playing within online platforms}

\begin{abstract}
RESUMEN
El co-playing en línea es una práctica de copresencia que conlleva la socialización entre personas ubicadas en distintos lugares físicos y que se ha incrementado en el período comprendido entre marzo y junio de 2020 , durante la crisis sanitaria de la COVID-19. Esta investigación tiene como objetivo entender las prácticas de co-playing en línea durante el confinamiento, en diferentes juegos y plataformas. Para ello, se ha utilizado el método de estudio de caso, con téc-

nicas etnográficas digitales: observación participante y entrevistas semi-estructuradas. El estudio ha identificado tres perfiles de practicantes del co-playing: iniciación, adaptación e intensificación. El primer perfil se caracteriza por haberse iniciado en estas prácticas dentro del contexto del confinamiento; el segundo ha adaptado sus prácticas lúdicas al espacio digital en línea. Mientras que, el tercero, ha incrementado el tiempo y la frecuencia de juego. Los resultados muestran que el co-playing ha permitido generar copresencia, tanto sincrónica como asincrónica, entre pares $y$, en menor medida, entre familiares. Finalmente, se destaca que la práctica del co-playing durante el confinamiento ha sido una actividad motivada por la búsqueda del bienestar, ya que ha ayudado a fortalecer lazos sociales y a contrarrestar el malestar y los sentimientos negativos producidos por el confinamiento y todas las consecuencias sociales asocia-
\end{abstract} das a la COVID-19

\section{PALABRAS CLAVE}

COVID-19; Co-playing; Copresencia; Prácticas sociales; Multijugador; Redes sociales.

\section{ABSTRACT}

Online co-playing is a practice of co-presence that allows people located in different physical places to socialize. This practice has increased during the period of lockdown (march and june of 2020) due to the COVID-19 health crisis. This study aims to understand online co-playing practices during the lockdown period, in various games and platforms. In this study, a case study methodology with digital ethnographic techniques was implemented: participant observation and semi-structured interviews. Three profiles of co-playing practitioners have been identified: initiation, adaptation and intensification. The first profile is characterized by having started to carry on these practices during the lockdown. The second corresponds to the adaptation of playful practices to online digital spaces. The third corresponds to the increment of time and frequency of play of those who already co-played online. The results show that co-playing online has allowed co-presence to occur, both synchronous or asynchronous, especially among peers, and to a lesser extent among family members. Thus, a generational gap has been detected. Finally, the practice of co-playing during the lockdown showed to be an activity motivated by achieving a wellbeing state, which has helped to strengthen social issues, to counter the distress and other negative feelings produced by the lockdown and all the social consequences associated with COVID-19.

\section{KEYWORDS}

COVID-19; Co-playing; Co-presence; Social practices;

Multiplayer; Social networks. 


\section{Les pràctiques de co-presència durant la COVID-19: co-playing en plataformes en línia}

\section{RESUM}

El co-playing en línia és una pràctica de co-presència que comporta la socialització entre persones ubicades en diferents llocs físics i que s'ha incrementat durant el període comprès entre el març i juny de 2020, durant la crisi del COVID-19. Aquesta investigació té com a objectiu entendre les pràctiques de co-playing en línia durant el confinament, en diversos jocs i plataformes. Per dur-la a terme, s'ha utilitzat el mètode de l'estudi de cas $a m b$ tècniques etnogràfiques digitals: observació participant $i$ entrevistes semi-estructurades. L'estudi ha identificat tres perfils de practicants del co-playing: el d'iniciació, el d'adaptació i el d'intensificació. El primer perfil es caracteritza per haver-se iniciat en aquestes pràctiques dins del context del confinament; el segon ha adaptat la seva pràctica lúdica a l'espai digital en línia $i$, el tercer, ha incrementat el temps i la freqüència de joc. Els resultats mostren que el co-playing ha permès generar co-presència, tant sincrònica com asincrònica, especialment entre iguals $i$, en menor mesura, entre familiars. Finalment, es destaca que la pràctica del co-playing durant el confinament ha estat una activitat motivada per la recerca del benestar, ja que ha ajudat a enfortir vincles socials i a contrarrestar el malestar i els sentiments negatius produits pel confinament $i$ totes les conseqüències socials associades al COVID-19.

\section{PARAULES CLAU}

COVID-19; Co-playing; Co-presència; Pràctiques socials; Multijugador; Xarxes socials.

\section{Introducción}

La crisis sanitaria de la COVID-19, ha puesto en evidencia que las formas de socialización no sólo se desarrollan dentro de los límites físicos y geográficos, sino que, cada vez más, lo hacen a través de plataformas y dispositivos digitales que nos permiten sentirnos y estar presentes digitalmente. Desde hace algunos años se viene hablando de la era de la "conectividad" o de una sociedad plataformizada (Van Dijck, 2013; Van Dijck, Poell y de Wall, 2018), donde cada vez más, nuestras prácticas sociales son intrínsecas al mundo digital.

En este artículo, exploramos la dinámica de la copresencia durante la crisis sanitaria de la COVID-19 por medio de las prácticas del co-playing (jugar y socializar con pares) que, justamente por la pandemia, han tomado especial relevancia en muchas de las esferas de la vida social. En este sentido, entendemos la copresencia digital a partir de las prácticas de co-visionado conectado (Pires y Roig, 2016), es decir, como prácticas que permiten la socialización entre personas que pueden estar ubicadas físicamente en distintos lugares pero que, al mismo tiempo, hacen uso de tecnologías y plataformas digitales para comunicarse y realizar actividades sincrónicas. Dichas prácticas, a su vez, permiten generar un sentimiento de presencia, similar a un espacio físico compartido. Con estas consideraciones, la pregunta de investigación que guía este estudio se formula de la siguiente manera: ¿cómo se llevaron a cabo las prácticas de co-playing durante la crisis sanitaria de la COVID-19?

Para llevar a cabo la investigación, hemos implementado la metodología de estudio de caso con métodos etnográficos digitales (Pires, 2018), durante los meses del confinamiento, es decir, entre marzo y junio del 2020. Concretamente, se ha llevado a cabo una observación participante y entrevistas semi-estructuradas online.

El estudio ha identificado tres perfiles de practicantes del co-playing: el de iniciación, el de adaptación, y el de intensificación, que, en conjunto, comparten la necesidad de establecer algún tipo de copresencialidad debido al período forzoso de distanciamiento social. En este contexto, la práctica del co-playing ha sido modelada por elementos y artefactos tales como las plataformas digitales y sus diseños, así cómo por la conexión a internet y las aplicaciones de mensajería instantánea.

\section{Copresencia Digital y Co-playing}

Las prácticas de copresencia digital han sido especialmente estudiadas en contextos de familias transnacionales y de migración (Alinejad, 2019). En la 
gran mayoría de ellas, se ha destacado el desarrollo de redes sociales y aplicaciones que permiten la comunicación sincrónica, difuminando las barreras entre la ausencia y la presencia. Además, esto ha facilitando la comunicación entre miembros de una misma familia que, aún en continentes diferentes, han podido sentir compañía (copresencia), y establecer o fortalecer las relaciones cercanas, demostrando que las plataformas digitales y las redes sociales tienden a aumentar la sensación de pertenencia (Baldassar et al., 2016). Alinejad (2019) ha detectado que las llamadas de voz representan una de las formas de comunicación más inmersivas de la copresencia. No obstante, las redes sociales y otras formas de comunicación también pueden retroalimentar la sensación de presencialidad y/o reforzar algunas normas físicas (pre)establecidas en un círculo social determinado.

Dentro de los estudios de copresencialidad, destacan las prácticas de co-playing, mayormente focalizadas en la relación entre el videojuego y la estructura familiar. Sin embargo, su enfoque principal está en las relaciones establecidas entre los jóvenes y sus familiares durante épocas de convivencia, en torno a las prácticas de juego. (Wang, Taylor y Sun, 2018; Chambers, 2012). En estos estudios la práctica de jugar conjuntamente (co-playing) representa, para la familia, una oportunidad de fortalecer sus vínculos (Walker, Brocato, Carlson y Laczniak, 2018). Este tipo de estudios se han abordado desde una perspectiva social pero, sobre todo, psicológica, sin considerar la posibilidad relacional que propicia la red, más allá de la presencia física de las personas en una misma estancia (Shin y Huh, 2011). En este sentido, existen escasos estudios que tratan sobre la importancia del co-playing más allá de las relaciones familiares en el ámbito del videojuego.

En algunos casos, las prácticas de co-playing forman parte de la modalidad de multijugador en línea. Los juegos multijugador permiten que diferentes personas interactúen en escenarios virtuales de manera simultánea, vinculando así las diferentes localizaciones físicas y, a su vez, posibilitando a los jugadores participar en procesos de socialización derivados del espacio ficcional (Egenfeldt-Nielsen, Smith y Tosca, 2019). El co-playing presenta características particulares pues, para que tenga lugar, se requiere la socialización con otros jugadores, ya sea de forma presencial o por medio de actividades en línea (Tiong Wee, 2015); mientras que, cuando se trata de un juego del tipo 'multijugador', las condiciones y características propias de estas plataformas, aunque puedan incluir herramientas para la socialización, posibilitan prescindir de una interacción social entre los jugadores, obviando así la característica de socialización del co-playing. En otras palabras, en esta investigación entendemos que el co-playing puede ser una característica de los juegos multijugador, pero no una condición obligatoria en ellos.

La situación derivada de la crisis sanitaria de la COVID-19 ha traído consigo un conjunto de prácticas digitales para facilitar el acompañamiento y las relaciones profesionales y personales en tiempos de pandemia. De acuerdo con Wijman (2020), a partir de la crisis de la pandemia, se han acelerado las tendencias en el uso y práctica de los videojuegos. De hecho, los (video)juegos han representado una forma de entretenimiento, escapismo e incluso socialización que se ha visto reflejado en el crecimiento exponencial de la industria (Wijman, 2020). En este contexto, el videojuego ha emergido como un medio que, aunque en buena parte consolidado, ha generado y ampliado nuevos espacios y prácticas de socialización como el co-playing, entre diferentes colectivos y contextos, en un momento de especial trascendencia social y tecnológica. Por este motivo, el presente estudio pone el foco en examinar cómo se han llevado a cabo las prácticas de co-playing durante la crisis sanitaria de la COVID-19 entre personas jóvenes, de diferentes partes del mundo.

\section{Teoría de Prácticas Sociales: un enfoque teórico- analítico}

Se ha utilizado el enfoque teórico-análitico de la segunda ola de prácticas sociales (Postill, 2010), que sostiene que a través del análisis de diferentes prácticas se puede comprender el orden social y la acción (Schatzki, 1996). El primero se entiende como la organización y relación que existe entre determinadas entidades (personas, instituciones, tecnología, materialidad etc.), y el orden que se establece entre todas ellas. Mientras que el segundo se entiende cómo la condición que posibilita la existencia de dicho orden social. El enfoque analítico sostiene que un fenómeno social puede propiciar un escenario donde se llevan a cabo un conjunto de prácticas sociales (Hui, Schatzki y Shove, 2017), como es el caso de la crisis de la COVID-19, que ha generado la necesidad de desarrollar prácticas de copresencia digital.

Desde este enfoque, las prácticas sociales se pueden entender desde dos perspectivas: como una entidad coordinada y como una performance (Schatzki, 1996; Reckwitz, 2002). La práctica, como una entidad coordinada, es abordada desde la vertiente organizativa de la misma, teniendo en cuenta sus normas y reglas, con el fin de ejecutarse. Dicha perspectiva, también incluye motivaciones, proyectos, tareas, propósitos, creencias, emociones y estados de ánimo para poder realizar una práctica social. Mientras que la práctica como una 
performance, corresponde a la acción humana y no humana, lo cual incluye la materialidad de los artefactos utilizados, y los lugares y tecnologías que posibilitan su existencia (Schatzki, 2015). Esto también incluye a las personas practicantes que pueden transformar sus prácticas a través de diferentes acciones, ritmos, rutinas, entre otras. Por tanto, este enfoque resulta apropiado para identificar y examinar cómo fueron realizadas las prácticas de copresencia digital a través del co-playing durante la crisis sanitaria de la COVID-19, a la vez que contribuye a entender el contexto de esas prácticas, las motivaciones de las personas practicantes y el por qué de las tecnologías utilizadas.

\section{Metodología}

Esta investigación se llevó a cabo entre marzo y junio de 2020, por el método de estudio de caso, con herramientas de recolección de datos de corte etnográfico en línea (Pires, 2018; Stake, 2005): observación participante, notas de campo y entrevistas semi-estructuradas.

Las investigadoras realizaron observación participante online con pequeños grupos, de máximo tres personas, durante una hora aproximadamente, y tomaron notas de campo mientras participaban activamente de las prácticas de co-playing. Además, se realizaron cinco entrevistas semi-estructuradas online, a través de apps de videollamada y mensajería instantánea, tales como WhatsApp y Messenger.

En las entrevistas se exploraron las motivaciones para jugar online con otras personas (co-playing), con quién(es) se ha jugado y las razones para jugar con las personas mencionadas. Durante la entrevista, también se consultó si el co-playing era una práctica social habitual, para las personas participantes, o si había emergido durante el período de confinamiento de la COVID-19. Así mismo, se indagó al respecto de las plataformas utilizadas para llevar a cabo el co-playing y las razones de su elección. Finalmente, se realizó una aproximación hacia el(los) significado(s) de esta práctica en el día a día de las personas participantes, frente a un contexto de crisis.

En este caso de estudio participaron trece personas: ocho hombres y cinco mujeres, entre 26 y 33 años. Las personas participantes estaban ubicadas en Brasil, Ecuador, España e Irlanda, quienes pertenecen a tres grupos de nacionalidad: brasileña, ecuatoriana y española.

El muestreo se hizo con la técnica de bola de nieve (Noy, 2008), con el fin de acceder a una práctica que, normalmente, se establece en canales de comunicación cerrados como apps de mensajería instantánea. Dicha técnica permitió establecer una muestra con personas de confianza, estableciendo una relación sólida y facilitando la obtención del consentimiento informado de las mismas.

Los casos estudiados siguieron los preceptos del caso instrumental (Stake, 2005), examinándose de manera individual para obtener información sobre un tema concreto. Así, aunque los sujetos o casos son de interés secundario, facilitan la comprensión de un fenómeno, que en el caso de este estudio, es la copresencia digital a través del co-playing durante la crisis de la COVID-19.

En el apartado de análisis y discusión se hace uso de pseudónimos, a fin de precautelar la integridad de las personas participantes.

\section{Análisis y discusión}

En este apartado analizamos y discutimos tres ejes fundamentales que han emergido durante el estudio de las prácticas de copresencia a través del co-playing en línea. La finalidad ha sido comprender cómo se han llevado a cabo este tipo de prácticas durante la crisis sanitaria de la COVID-19, teniendo en cuenta los perfiles de sus practicantes, el rol de las plataformas, el tipo de juego y dispositivos tecnológicos y, finalmente, las personas con las que se ha compartido estas prácticas.

\subsection{Perfiles de Co-playing: Iniciación, Adaptacióne Intensificación}

A partir de la observación participante y de las entrevistas semiestructuradas, fue posible identificar tres perfiles de practicantes del co-playing durante la crisis de la COVID-19. Éstos han sido denominados como: a) iniciación; b) adaptación; y c) intensificación. El primer perfil reúne a todas aquellas personas practicantes que no jugaban antes la pandemia. El segundo perfil, en cambio, se refiere a aquellas personas que realizaban esta práctica, especialmente con juegos de mesa y que, a raíz del confinamiento, adaptaron sus juegos a diferentes espacios digitales en línea. Finalmente, el tercer perfil incluye a aquellas personas que ya jugaban en línea con sus pares, pero que, a partir de la situación de crisis, incrementaron sus prácticas de una manera más intensa.

La iniciación en el co-playing digital ha tenido diversas motivaciones. Para Marcos (29 años, hombre brasileño, ubicado en Brasil), que reconoce preferir una interacción directa al momento de realizar sus prácticas de juego, el co-playing online le ha servido para compartir momentos con sus amigos y, a la vez, canalizar sus 
emociones: "siempre he preferido las interacciones en persona, pero para mí fue una manera de aligerar el día y tuvo un efecto terapéutico frente al caos." La necesidad que señala Marcos, de escapar del caos a través del co-playing, ha sido identificada anteriormente como parte integral de un jugador/a que necesita escapar de su realidad, es decir, que utiliza el juego como una forma de catarsis (Vilasís-Pamos y Pires, próximamente).

En la misma línea, María (30 años, mujer ecuatoriana, ubicada en Ecuador), también destacó su necesidad de desconectar. Pese a que su iniciación en el co-playing fue propiciada por una amiga, a través de una invitación por Facebook, los elementos de desconexión de la realidad están presentes cuando ella reconoce la importancia de mantener el contacto con su círculo social en medio de la situación de confinamiento:

"Fue mi primera experiencia y la considero la más importante porque fue a larga distancia, cada una [de las amigas] en un sitio y horario distinto. [El co-playing] me ayudó a salir de la rutina y a socializar en medio de toda la situación que vivimos. No tenía más opciones, así que socializar a través de un juego hizo que al menos conversara un poco".

A esta experiencia se suma la de Célia (33 años, mujer brasileña, ubicada en Irlanda), a quien la nueva práctica del co-playing le proporcionó un alivio del estrés que provocaba la situación y la ayudó a acercarse a sus amigos que viven lejos. De este modo se pone en evidencia que, las prácticas de co-playing han tenido lugar porque sus practicantes buscan en ellas una forma de bienestar, que proporciona cierto alivio a la situación derivadas de la crisis de la COVID-19.

La distancia es un factor clave a considerar dentro de la práctica del co-playing en línea pues supone, por un lado, acortar las distancias al mantener un contacto social por medio del juego, y, por otro, un catalizador de emociones negativas, tales como el estrés y la ansiedad. En este sentido, muchas de las prácticas de juego, frente a la situación de crisis y confinamiento, también han adaptado sus funcionalidades para permitir la continuidad de sus comunidades. Así, algunas de éstas, que tradicionalmente han requerido de un contacto presencial y se han basado en dinámicas de juego de mesa, como Magic, mudaron su comunidad a un entorno virtual en el que, de alguna manera, se han mantenido conectados. Así lo comentó Francisco (27 años, hombre ecuatoriano, ubicado en España): "Magic, que es básicamente un juego físico (de encuentro) se pasó a lo digital y ya no había la parte social, que era lo que más nos movía. Pero he amanecido jugando con amigos con quienes conversamos." En su reflexión, Francisco comentó que las limitaciones y diferencias que le supuso este cambio en medio de la crisis ha hecho que su cerebro actúe de manera diferente. Para Paula (26 años, mujer española, ubicada en España), las prácticas de co-playing han sido de pocas horas y con la finalidad de divertirse, pero también de olvidar la realidad de la pandemia, que se traduce en la necesidad de "olvidar el creciente número de defunciones diarias y el bombardeo constante de noticias relacionadas con la COVID-19".

Como señalan muchas de las respuestas, el cambio de prácticas hacia el co-playing se determinó en buena medida por las restricciones que mermaron los encuentros sociales. En el caso de Carolina (31 años, mujer ecuatoriana, ubicada en España), el cambio de sus prácticas habituales de juegos de mesa y cartas, a una modalidad online, supuso un beneficio a la hora de evaluar la situación del confinamiento pues, al estar en casa, de alguna manera los juegos online facilitaban el contacto con sus amigas y familiares en Ecuador, incluso rescatando prácticas y juegos cuyas emociones positivas sirvieron de aliciente dentro del período de confinamiento.

\begin{abstract}
"Al estar en casa por mucho más tiempo, siento que pude acompañar el confinamiento de mis familiares y amigos en Ecuador. En un momento sentí la necesidad de desconectar de toda la información que me agobiaba y, por medio de juegos lúdicos, me sentí cerca de mis seres queridos. ¡Volvimos a jugar parchís!"
\end{abstract}

No obstante, la dinámica del co-playing no resultó como algo nuevo o de adaptación para todos los participantes de este estudio pues, en el caso de aquellas personas que tenían una práctica más o menos regular de co-playing, el confinamiento les supuso, en gran medida, una oportunidad para intensificar las horas de juego. Así lo menciona Ricardo (29 años, hombre ecuatoriano, ubicado en España), para quien la cuarentena le ha servido como excusa para practicar co-playing aún más:

"Lo hacía [co-playing] y lo hago mucho más seguido
a partir de haber venido a vivir a España porque es
mi forma de mantener el contacto con mis amigos.
Considerando la cuarentena, esto ha significado una
forma de no perder las relaciones sociales, sí. Pero,
sobre todo, ha sido una forma de mantenerme dis-
traído. Muchas personas no sabían qué serie ver o
qué libro leer, pero para nosotros, los gamers, es
meternos en la casa a jugar y nunca nos aburrimos."

Para el grupo de participantes del tipo 'intensificación', la crisis del coronavirus se vivió de una manera atípica. En el caso del grupo de hombres españoles ubicados 
en España, conformados por Lluc (29 años), Bernat (26 años), Álex (27 años) y Arnau (27 años), ninguno de ellos demostró especial preocupación por la COVID-19, aunque sí por la situación generada y las posibles implicaciones para sus familias y amistades. Este grupo en particular, practica el co-playing con el juego League of Legends - (LoL). El incremento del colectivo involucrado en la práctica del co-playing lo atribuyen al hecho que LoL es un juego de libre acceso y sin requerimientos técnicos. Además, Lol permite la comunicación hablada a través de la propia plataforma, sin coste añadido.

Sin embargo, de acuerdo con el grupo entrevistado, una característica de Lol es que el hecho de jugar con el ordenador supuso la incorporación de más personas en el grupo de co-playing. No obstante, también imposibilitó la participación de otro compañero con el que anteriormente compartieron momentos de co-playing con la Playstation 4 (PS4), pero éste no jugó a LoL porque no tuvo ordenador. Esto es relevante para el estudio en el sentido que, al hablar de co-playing en línea, es indispensable contar con la tecnología y los medios adecuados, como lo detallaremos más adelante. Los jugadores de Lol coincidieron, finalmente, en que la práctica de co-playing ayudó a evadir su realidad, a modo de oasis cotidiano y punto de encuentro con sus amigos.

\subsection{Las plataformas, juegos y canales de comunicación}

Como cualquier otra práctica social, el co-playing online necesita artefactos, materiales y tecnologías para poder ser llevado a cabo (Schatzki, 2015). Tanto los artefactos que se utilizan, así como las personas que lo practican pueden moldear la forma en que dicha práctica ocurre (Pires, Masanet y Scolari, 2019). Por ello, además de la motivación de los jugadores, las prácticas de co-playing en línea, en tiempos de confinamiento, requirieron elementos como una buena conexión a internet, plataformas que permitan jugar conjuntamente a determinados juegos y canales de comunicación para poder realizar la copresencia en estos espacios digitales.

Además de saber jugar, los tres perfiles de co-playing identificados comparten la necesidad de contar con las herramientas para jugar y compartir este momento, aunque la interacción no sea siempre completamente sincrónica. Por ejemplo, en uno de los grupos en el que se participó, se jugó Stop, un juego que permite crear categorías de elementos que deben ser completadas en un determinado período de tiempo, a partir de que dichos elementos inicien con la misma letra del alfabeto que se determina previamente. Célia (33 años, brasileña, ubicada en Irlanda) jugó el juego Stop, en dos plataformas diferentes. En una de las plataformas (StopotS),
Célia compartió su práctica con una de las investigadoras y con otro participante, donde pudieron establecer conversaciones a través de un chat, propio de la plataforma. No obstante, debido a que el ritmo del juego suele ser muy rápido, la regla temporal puede establecerse, por ejemplo, en cinco minutos. De esta manera, se unifica el tiempo para rellenar las categorías para todos los participantes. La excepción, sin embargo, está definida cuando alguna de las personas jugadoras termina antes del tiempo definido y, por lo tanto, concluye la partida. Es, en este punto, en que el aplicativo del chat de la plataforma resultó deficiente para mantener una copresencialidad de calidad, pues se requería focalizar la atención en rellenar las categorías en muy poco tiempo, por lo que, una llamada, a través de Whatsapp, dio solución eficaz. En esta dinámica, Célia también señala que los problemas de conexión con el juego, la obligó al cierre forzado y a la consecuente pérdida de puntos al volver a incorporarse a la partida. Aún así, ella insiste que no ha supuesto una decepción ni un motivo para no seguir jugando, pues para ella la copresencialidad significó compartir momentos con sus amigos y disminuir la sensación de ansiedad. Tal cómo sucedió con el uso de Whatsapp (como alternativa sincrónica), también se mudó el juego a la plataforma StopS, que permite el co-playing asincrónico: "optamos por la plataforma StopS porque estábamos ubicados en continentes diferentes y aún así podíamos jugar unos contra los otros sin necesidad de estar todos online al mismo tiempo". Célia insistió en que en muchas ocasiones utilizaron Whatsapp, aunque fuera para enviar un mensaje y comentar el juego por notas de voz. De esta manera, pudo sentirse un poco más acompañada por sus amigos durante el confinamiento.

En otro de los grupos en los que participamos, se realizó el co-playing con el juego de Mario Kart, en la consola Nintendo Switch, utilizando una aplicación propia de Nintendo, habilitada únicamente para smartphones, que permite realizar llamadas de voz. Sin embargo, y en relación a la casuística anterior, la app no estableció una buena conexión, ni permitió realizar una llamada de voz. Por esta razón, una vez más, se optó por utilizar Whatsapp como el canal de comunicación. Esto pone en evidencia que la materialidad disponible y accesible tiene una relación directa con las prácticas del co-playing en línea.

Cabe destacar, que Alan (29 años, hombre brasileño, ubicado en Brasil), poseedor de diferentes consolas como PS4, Nintendo Switch y Xbox, y con suscripciones para el juego online, afirmó que antes de la crisis sanitaria utilizaba las suscripciones para jugar en línea con personas desconocidas con la intención de retarse y autosuperarse, partida a partida. Aunque las subscrip- 
ciones lo permitían, Alan comentó que no conversaba durante sus partidas, porque no sentía la necesidad de la copresencia digital; por el contrario, prefería practicar el co-playing con sus amigos in-situ. Esto cambió durante la crisis, pues pasó "a jugar con amigos y hablar por Whatsapp para poder sentir que estaba en un espacio único de socialización" y para poder "crear buenas memorias de un período tan complejo".

En un caso parecido al de Alan, un grupo de amigas ubicadas en distintos puntos de España, optaron por utilizar Whatsapp como un elemento fundamental de la práctica, creando un grupo en la misma aplicación para poder realizar el co-playing. Dicho grupo sirvió como parte de la entidad coordinada de la práctica del co-playing y como un elemento de su performance, ya que fue utilizado como una herramienta de gestión de la práctica. De este modo, fue posible establecer la disponibilidad de cada jugadora, a la hora de iniciar la práctica, combinándola con sus rutinas y facilitando la conciliación profesional y familiar. Además de eso, el grupo creado en la app, fue el canal de comunicación durante sus partidas online.

Todos los casos expuestos evidencian que Whatsapp es un canal de comunicación muy utilizado entre las participantes del estudio, pues lo consideran fácil de utilizar. A lo que se suma el hecho de facilitar llamadas de calidad, con el valor adicional de que la mayoría de las personas lo tienen instalado en sus móviles.

Inevitablemente, las prácticas de copresencialidad se han visto moldeadas por la situación de crisis y, por lo tanto, el acceso a las plataformas también ha cambiado. El ejemplo de Paula (26 años, española, ubicada en España) ilustra esta situación. Previo a la crisis, solo llevaba a cabo prácticas de co-playing con su pareja y compañeras de piso, y, de manera particular, en el juego de móvil Clash of Clans. Unos meses antes de la pandemia, había practicado el co-playing con la Nintendo Switch para poder probar el mes de gratuidad que ofrece la suscripción online. Sin embargo, con la crisis, procedió a contratar la opción anual en previsión de necesitar la compañía, aunque virtual, de sus amigas. Para ella, el co-playing no solo ha significado evasión y diversión, sino también proximidad con sus amistades, a las que no podía ver debido a la situación generada por la pandemia. La incertidumbre y la (no) previsión a medio plazo, de un cambio considerable en las relaciones sociales, fue decisiva para empezar a realizar prácticas de co-playing virtual y contratar la suscripción anual de Nintendo. En dicho caso, la necesidad de pagar una suscripción online para poder realizar las prácticas de co-playing en línea puede tener un doble significado. Por un lado, la suscripción online es un elemento fun- damental para la práctica de co-playing, pero a la vez un elemento excluyente para todas aquellas personas que no pueden asumir su coste. Sin embargo, el pago es necesario para garantizar el mantenimiento del propio servicio y de la plataforma.

Momentos de crisis, como el actual, han puesto en evidencia la necesidad de contar con una infraestructura potente que permita la conexión simultánea de miles de usuarios. Durante una de las sesiones de co-playing, de hecho, surgió la idea de jugar a través de la plataforma llamada Board Game Arena, donde hay diferentes juegos de mesa. Sin embargo, solo un miembro del equipo estaba dado de alta en la plataforma y, debido al incontable número de solicitudes que recibió la plataforma durante los últimos meses, fue imposible realizar el registro del resto de participantes del grupo. Esta situación no solo demuestra la importancia del rendimiento tecnológico de la infraestructura de la plataforma, sino también cómo ésta moldea la (no)ocurrencia de la práctica y quien la performa.

\subsection{Las relaciones sociales en el co-playing}

Las prácticas de co-playing digital, en el período de la COVID-19, han demostrado ser inseparables de un conjunto de prácticas que aparentemente son dispersas pero que, cuando se juntan, facilitan el sentimiento y experiencia de la copresencia, aunque de forma digital.

Dichas prácticas pueden tener diferentes formatos: videollamadas, mensajes de texto y notas de voz con personas cercanas, por mencionar algunas de ellas. La gran proliferación de las plataformas, las aplicaciones y los canales de comunicación han facilitado la copresencia digital. Tal como señala el estudio de Alinejad (2019), las llamadas de voz son canales de comunicación que permiten la inmersión en la copresencia digital; y, en el presente estudio, también han resultado ser un elemento fundamental del co-playing en línea.

El co-playing, en gran medida, se llevó a cabo entre amigos, o con miembros de la familia, de la misma franja de edad. Joanna (32 años, brasileña, ubicada en España) jugó con sus amigas, residentes en su misma ciudad, y con su hermano de 36 años, que vive en Brasil. Ella comentó que decidió jugar con esas personas por su afinidad y habilidad con los videojuegos y el uso de plataformas digitales. Este hecho evidencia un posible sesgo generacional a la hora de realizar prácticas de co-playing en línea, de acuerdo con lo que han planteado las personas participantes de este estudio.

En la misma línea argumental, Célia (33 años, mujer brasileña, ubicada en Irlanda) explicó que la copresencia que mantiene con su familia se hace directamente 
con llamadas de vídeo, y que el co-playing lo hace con sus amigos de la misma franja etaria. Así, aunque la copresencia es una práctica habitual entre familias en situación migratoria, con el fin de mantener sus lazos afectivos, esta práctica también ha resultado relevante para el mantenimiento de las relaciones a corta distancia y entre pares, a través del co-playing en línea.

En resumen, las personas participantes en el estudio realizan co-playing en línea por su interés en juegos determinados, además de la necesidad de mantener contacto con otras personas, reafirmando así la importancia de las motivaciones, propósitos e intereses para llevar a cabo una práctica social (Schatzki, 1996).

\section{Conclusión}

A partir de los casos de estudio analizados, la investigación sostiene que existe una motivación general que acompaña la práctica del co-playing en línea, durante el tiempo de confinamiento. Esta motivación está más o menos generalizada en los tres perfiles identificados: 1) iniciación, 2) adaptación y 3) intensificación. Por un lado, se destaca la necesidad de aliviar la ansiedad y el estrés generados por la crisis; por el otro, entran en juego los sentimientos de compañía y cercanía que se presentan con mayor intensidad y están relacionados directamente con las relaciones sociales. Así mismo, se sostiene que la idea que une a algunas personas que practican el co-playing, en tiempos de confinamiento, es la de disminuir las prácticas de consumo de información e interactuar de manera más saludable y distraída con las personas más cercanas, especialmente, con aquellas con las que tienen un vínculo de afecto importante. Finalmente, en otros casos, como los perfiles de intensificación, se sostiene que sus prácticas se llevan a cabo de manera habitual y no sólo desde la evasión de la realidad informativa, sino por el mero hecho de entretenerse.

El comprender la práctica de co-playing como una actividad saludable es un punto importante a destacar en este estudio, pues tiene una relación directa con el fortalecimiento de los lazos sociales que, en una situación como la crisis de la COVID-19, es un elemento clave para contrarrestar el malestar y los sentimientos negativos derivados de las medidas restrictivas.

Las respuestas y dinámicas de las personas que han participado en este estudio, nos permiten sugerir que tanto el ingreso a las prácticas de co-playing, así cómo la adaptación o la intensificación de estas prácticas han permeado en una buena porción de la sociedad, particularmente en el escenario del confinamiento por la COVID-19 (Wijman, 2020). Por esta razón, sugerimos que, pese a no ser una práctica nueva, la emergencia y el confinamiento a partir de la COVID-19 ha supuesto un incremento y adaptabilidad mucho mayor en el ejercicio del co-playing.

En este sentido, también es relevante destacar que las prácticas de co-playing no se han restringido al mero uso de juegos y/o plataformas, sino que a éstas se han ido incorporado otros medios, ya conocidos, tales como Whatsapp, cuya finalidad (en cuanto al co-playing) es mejorar la comunicación dentro de la experiencia de juego, además de ser un elemento indispensable en la creación y el fortalecimiento de las relaciones sociales. Cabe destacar también que Whatsapp, y otras aplicaciones, permiten la comunicación sincrónica, que tiene cierto grado de predominancia cuando se realizan prácticas de co-playing, sobre todo por la interacción inmediata que estas proporcionan.

Pese a que hay algunos casos en los que las personas participantes reconocieron haber interactuado por medio de estas prácticas con sus familiares más cercanos (como hermanos y primos), la gran mayoría insiste en que la dinámica del co-playing la llevan a cabo, de manera más habitual, con sus grupos de pares, ya sean amigos y amigas más cercanas, así como colegas de la época del instituto, universidad y trabajo. Si bien la muestra del estudio corresponde a una franja de edad más o menos limitada, nos permite también reflexionar con respecto a las posibles diferencias que pudieran existir en la práctica del co-playing considerando el factor de la edad. En este sentido, puede ser interesante, para futuras investigaciones, que se tome en cuenta diferentes generaciones a fin de observar qué diferencias existen dentro de las actividades de co-playing, especialmente en momentos de crisis.

\section{Referencias}

Alinejad, D. (2019) Careful Co-presence: The Transnational Mediation of Emotional Intimacy. Social Media + Society, Online First, 1-11. https://doi.org/10.1177/2056305119854222

Baldassar, L., Nedelcu, M., Merla, L., y Wilding, R. (2016). ICT-based co-presence in transnational families and communities: challenging the premise of face-to-face proximity in sustaining relationships. Global Networks, 16 (2),133-144. https://doi.org/10.1111/glob.12108

Chambers, D. (2012). 'Wii play as a family': the rise in family-centred video gaming. Leisure Studies, 31(1), 69-82. https://doi.org/10.1080/0 2614367.2011.568065

Egenfeldt-Nielsen, S., Smith, J. H., y Tosca, S. P. (2019). Understanding video games: The essential introduction. Routledge.

Hui, A., Schaztki, T. R., y Shove E (2017). Introduction. En Hui A., Schaztki T. R., y Shove E. (eds.). The Nexus of Practices: Connections, constellations, practitioners. ( $p p-1-7)$. Routledge.

Noy, C. (2008). Sampling Knowledge: The Hermeneutics of Snow- 
ball Sampling in Qualitative Research. International Journal of Social Research Methodology, (11), 4, 327-344. https://doi. org/10.1080/13645570701401305

Pires, F., Masanet, M-J ., y Scolari, A. C. (2019). What are teens doing with YouTube? Practices, uses and metaphors of the most popular audio-visual platform. Information, Communication \& Society, Online, 1-17. https://doi.org/10.1080/1369118X.2019.1672766

Pires, F. (2018). Researching co-viewing on social media and instant messaging applications: ethics and challenges. Comunicação e Sociedade, 33(2), 409-424. http://revistacomsoc.pt/index.php/comsoc/ article/view/2924

Pires, F., y Roig, A. (2016). Challenging prime time television: Co-viewing practices in the Brazilian telenovela. Convergence: The International Journal of Research into New Media Technologies, 22(4): 392-407. https://doi.org/10.1177/1354856516641623

Postill, J. (2010) Introduction: Theorising Media and Practice In Braüchler, B, y Postill, J. (eds.) Theorising media and practice. (pp. 1-34). Berghahn Books.

Reckwitz, A. (2002). Toward a theory of social practices: A development in culturalist theorizing. European Journal of Social Theory, 5(2), 243-263. https://doi.org/10.1177/13684310222225432

Schatzki, T. R. (2015). Spaces of Practices and of Large Social Pheno- mena. EspacesTemps.net, Travaux. http://www.espacestemps.net/ articles/spaces-of-practices-and-of-large-social-phenomena/

Schatzki, T. R. (1996) Social Practices: A Wittgensteinian Approach to Human Activity and the Social. Cambridge University Press.

Stake, R. E. (2005). Qualitative case studies. En Denzin, N. K ., y Lincoln, Y.S. (Eds.), The Sage handbook of qualitative research (3rd ed.), (pp. 443-466). Sage.

Shin, W., y Huh, J. (2011). Parental mediation of teenagers' video game playing: Antecedents and consequences. New Media \& Society, 13(6), 945-962. https://doi.org/10.1177/1461444810388025

Tiong Wee, S. (2015). Understanding the learning and social gameplay experience of older adults playing a Bingo digital game [Tesis doctoral. Simon Fraser University]. https://summit.sfu.ca/item/16066

Van Dijck, J. Poell, T., y de Wall, M. C. (2018). The platform society: public values in a connected world. Oxford University Press.

Van Dijck, J. (2013) The culture of connectivity: A critical history of social media. Oxford University Press.

Vilasís-Pamos y Pires. (s/f). How do teens define what it means to be a gamer? Mapping teens' video game practices and cultural imaginaries from a gender and sociocultural perspective. [En revisión].

Walker,D., Brocato, E.D., Carlson, L., y Laczniak, R.N. (2018). Parents' POSICIONAMIENTO EN BUSCADORES?

Observatorio de Cibermedios

\section{https://observatoriocibermedios.upf.edu/}

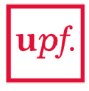

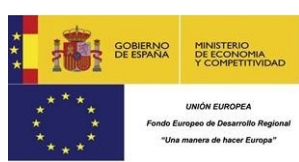

El Observatorio de Cibermedios es una producción del Grupo de Investigación en Documentación Digital y Comunicación Interactiva (DigiDoc) del Departamento de Comunicación de la Universitat Pompeu Fabra.

El Observatorio de Cibermedios (OCM) forma parte del proyecto del Plan Nacional "Narración interactiva y visibilidad digital en el documental interactivo y el periodismo estructurado". RTI2018-095714-B-C21 (MINECO/FEDER), Ministerio de Ciencia, Innovación y Universidades (España). 
and children's violent gameplay: role of co-playing. Journal of Consumer Marketing, 35(6):623-632. https://doi.org/10.1108/JCM-102017-2397

Wang, B., Taylor, L., y Sun, Q. (2018). Families that play together stay together: Investigating family bonding through video games. New Media \& Society, 20(11), 4074-4094. https://doi. org/10.1177/1461444818767667

Wijman, T. (2020). Global Game Revenues Up an Extra \$15 Billion This Year as Engagement Skyrockets. Newzoo. https://newzoo. com/insights/articles/game-engagement-during-covid-pandemic-adds-15-billion-to-global-games-market-revenue-forecast/?utm_campaign=GGMR\%202020\&utm_source=email\&utm_ medium=GGMR\%20Nov

\section{CV}

Fernanda Pires. Es investigadora Juan de la Cierva en el grupo de investigación MEDIUM, del Departamento Comunicación de la Universitat Pompeu Fabra, UPF, de Barcelona. Es doctora con mención internacional en Sociedad de la Información y el Conocimiento por la Universitat Oberta de Catalunya. Ha realizado estancias de investigación en Brasil ( USP - Universidade de São Paulo) y Australia (RMIT- Royal Melbourne Institute of Technology). Investiga los usos y las prácticas sociales llevadas a cabo con/en las plataformas digitales interactivas por parte de sus usuarios, y cómo ellos se relacionan con las lógicas impuestas por las mismas. También investiga educación mediática informal a través de prácticas creativas. Es experta en métodos digitales cualitativos y prácticas de copresencia digital como el covisionado conectado.

Júlia Vilasís-Pamos. Es Estudiante del Doctorado en Comunicación y miembro del Grupo de Investigación MEDIUM del Departamento de Comunicación, UPF. Está graduada en Ciencias Políticas y de la Administración (UPF, 2016) y ha cursado el Máster en Estudios Internacionales de Poder, Medios y Diversidad (UPF, 2018). Actualmente está trabajando en su tesis doctoral sobre la construcción de la identidad gamer a través del género y la clase social. Sus principales líneas de investigación son los estudios del videojuego, el género, la clase social, la cultura popular y los estudios sobre juventud.

Priscila Alvarez-Cueva. Es estudiante de doctorado en el Departamento de Comunicación de la Universidad Pompeu Fabra. Es miembro de dos grupos de investigación, CRITICC y JOVIScom, en la UPF. Actualmente está trabajando en su tesis doctoral sobre música comercial y su papel en la construcción de identidades entre adolescentes y adultos jóvenes, en España y Ecuador. Tiene una licenciatura en comunicación social y publicidad de la Universidad del Azuay (Cuenca, Ecuador), y una maestría en estudios internacionales, medios, poder y diferencia de la Universidad Pompeu Fabra. En 2020, realizó una visita de investigación en la Universidad de Oporto.

Ona Anglada-Pujol. Es estudiante del Doctorado en Comunicación en el Departamento de Comunicación, UPF, vinculada al grupo de investigación MEDIUM. Está graduada en Comunicación Audiovisual (UPF, 2016) y ha realizado el Máster Universitario en Investigación en Comunicación Social (UPF, 2019). Su tesis doctoral se centra en las prácticas de slash fiction del fandom de los gamers y los discursos sobre género, sexualidad y amor romántico, así como la relación que establecen los propios youtubers con estos textos. Sus líneas de investigación son los estudios de género y queer, los fan y celebrity studies. 